\section{Cureus}

Received 06/02/2013

Review began 06/03/2013

Published 08/19/2013

\section{(c) Copyright 2013}

Leung et al. This is an open access article distributed under the terms of the Creative Commons Attribution License CC-BY 3.0., which permits unrestricted use, distribution, and reproduction in any medium, provided the original author and source are credited.

\title{
Cost Minimization Analysis: Should Partial Breast Irradiation Be Utilized Over Whole Breast Irradiation Assuming Equivalent Clinical Outcomes?
}

\begin{abstract}
Martin Leung ${ }^{1}$, Michael Lock ${ }^{2}$, Alexander Louie ${ }^{3}$, George Rodrigues ${ }^{3}$, David D'Souza ${ }^{4}$, Robert Dinniwell ${ }^{5}$, Rob Barnett 6

1. University of Western Ontario 2. Department of Radiation Oncology, London Regional Cancer Program, London, Ontario, CA; Schulich School of Medicine \& Dentistry, Western University, London, Ontario, CA. 3. Department of Radiation Oncology, London Regional Cancer Program, London, Ontario, CA; Schulich School of Medicine \& Dentistry, Western University, London, Ontario, CA 4. Department of Radiation Oncology, London Regional Cancer Program, London, Ontario, CA 5. Cancer Clinical Research Unit (CCRU), Princess Margaret Cancer Centre 6. Department of Medical Physics, London Regional Cancer Program, London, Ontario, CA
\end{abstract}

$\square$ Corresponding author: Martin Leung, mleung2014@meds.uwo.ca Disclosures can be found in Additional Information at the end of the article

\section{Abstract}

Introduction: External beam partial breast irradiation (EB-PBI) is being used more frequently as an alternative to whole breast irradiation (WBI) in the adjuvant treatment of early-stage breast cancer. Breast cancer represents a substantial proportion of the workload for cancer centres; therefore, EB-PBI represents a possible alternative treatment of equal effectiveness that can have a significant impact on costs and patient throughput. However, planning this therapy requires increased quality assurance and resource allocation. Therefore, a cost minimization analysis was performed to compare WBI versus EB-PBI. Based on this study, recommendations on appropriate resource allocation and cost of resources at each step of planning can be made to maximize cost efficiency.

Materials and Methods: Cost minimization requires a detailed determination of resource utilization for each of the two treatments. Activity-based costing was used to create a model of radiotherapy costs. A process map was developed that separated the management of patients into differentiated quantifiable units (dosimetry, QA, active treatment, other preparatory work). Time, labour costs, and capital costs were measured using interviews and validated with timed analyses. The perspective of the analysis was that of the hospital budget at a comprehensive cancer clinic in Canada. Thus, personal patient costs and radiation oncologist labour costs were not included in the analysis as these are not funded by the hospital budget. The WBI regimen was 50.0Gy in 2.0Gy fractions, taking place over five weeks. The EBPBI was 38.5Gy in 3.85Gy fractions twice per day for five days. The two treatment arms are considered to have equivalent clinical outcomes.

Results: The total costs per patient for WBI and EB-PBI were 1346.20 Canadian dollars (2012) and 1128.70 Canadian dollars, respectively. The capital costs per patient for WBI and EB-PBI were 937.50 Canadian dollars and 721.88 Canadian dollars, respectively. Labour costs accounted for $30 \%$ of WBI and 36\% of EB-PBI. EB-PBI was 19\% less expensive than WBI. Per patient, this is a cost difference of $\$ 217.50$, or savings of $\$ 21,562.50$ based on the department workload of 100 breast cancer patients per linear particle accelerator per year. The majority of the cost differences arose from both capital and labour costs needed for the extra fractions per patient 
required for WBI.

Conclusions: EB-PBI significantly minimizes costs in the treatment of early-stage breast cancer relative to WBI. These results may be utilized by other institutions with other similar health care systems when executing decisions regarding resource allocation in the context of earlystage breast cancer treatment. Costs can be adjusted for each activity within the model. In addition, changes in operating parameters can be adjusted allowing other centres to determine detailed cost impacts specific to their own centre. The model can also be applied to different disease treatment methods.

Categories: Medical Physics, Radiation Oncology, Miscellaneous

Keywords: breast cancer, cost minimization analysis, partial breast irradiation, whole breast irradiation, irradiation

\section{Introduction}

Currently, the standard-of-care for early-stage breast cancer continues to be lumpectomy followed by adjuvant radiotherapy. This was demonstrated by the National Surgical Adjuvant Breast and Bowel Project (NSABP) B-06 trial, which showed that lumpectomy followed by whole breast irradiation (WBI) resulted in similar survival outcomes to modified radical mastectomy [1]. Unfortunately, the use of conventionally fractionated whole breast irradiation (CF-WBI) incurs several disadvantages, including a lengthy treatment time (five to seven weeks). Additionally, most tumor recurrences occur at the site of the lumpectomy cavity [2], but the current standard is delivery of radiation beyond the lumpectomy bed. This results in an increased dose to critical surrounding structures, including the lungs and heart [3].

This led to the development of accelerated partial breast irradiation (APBI), which drastically reduces treatment time while treating only part of the breast, leading to improved convenience and possibly morbidity [4]. The American Society for Radiation Oncology (ASTRO) consensus statement discussed APBI's increase in the risk of ipsilateral breast tumor recurrence (IBTR) associated with an increased risk for distant metastasis and death, as well as an increased risk of toxicity, including local fibrosis or poor cosmesis [5]. However, a recent study by Ferraro, et al. showed no significant difference between APBI and WBI for local recurrence rate, disease-free survival, cause specific survival, and overall survival at five years [6]. In addition, Vicini, et al. applied the ASTRO Consensus Panel (CP) levels of suitability ("suitable", "cautionary", and "unstable") for the use of APBI to a large group of patients at their institution and found no difference in the risk of IBTR between the three groups [7]. The three modalities of APBI commonly utilized include external beam (EB-PBI), interstitial brachytherapy (IB-PBI), and balloon catheter brachytherapy (MS-PBI). The NSABP B-39/Radiation Therapy Oncology Group (RTOG) 0413 Phase III trial, initiated in 2006, randomizes patients to either WBI or any of the three PBI modalities. A similar trial, the RAPID trial, randomized patients to either WBI or 3DCRT APBI. Although data on equivalence of APBI and WBI currently comes from trials with shorter follow-up and fewer patients [8-10], this technique has become increasingly popular with patients, hospitals, and health care providers. In addition to patient convenience, there is also an underlying assumption that it is less costly [11]. However, this has not been clearly established, especially in the Canadian context.

As this treatment technique is increasingly utilized in the management of early-stage breast cancer, it is important to consider its impact on healthcare costs. There have been two cost comparison studies published in the United States comparing APBI to WBI, concluding that EBPBI was the most cost-effective strategy among the PBI modalities [12-13]. To our knowledge, there have been no publications in Canada comparing costs of the two treatments. 
The purpose of this study is to evaluate resource utilization of WBI versus EB-PBI in the treatment of early-stage breast cancer using a cost minimization analysis. This type of cost comparison is based on the presumption that EB-PBI and WBI produce equivalent clinical outcomes, and, in accordance with the consensus statement from ASTRO, that PBI can be acceptable as an alternative outside of a clinical trial in appropriate patient groups [5]. The objective of this study is to determine if EB-PBI or WBI incurs the least cost.

\section{Materials And Methods}

This study compares the relative costs associated with WBI or EB-PBI in the context of the hospital budget at our institution, in the form of cost-minimization analysis model. Only direct costs are taken into account, which include labour and capital costs directly associated with radiation treatment. Other costs excluded from our analysis include non-operating budget costs (radiation oncologist salaries), base costs for all patients (administration, information technology (IT), information systems (IS), building costs, facility costs, and procurement), and operating budget costs (linear accelerator (linac) servicing and parts). Patient out-of-pocket costs, such as parking and loss of income for time-off work, are not included.

Both treatment regimens are implemented post-lumpectomy in early-stage breast cancer patients (Stages 0, I, or II). The ASTRO Consensus Statement indicated that selection outside of a clinical trial include all patients that are "candidates for breast-conserving (no prior radiotherapy, no history of collagen vascular diseases, and not pregnant)". In addition, the committee indicated a 'suitable' group for APBI with criteria, such as age greater than or equal to 60 , tumour, $\mathrm{T} 1$ stage, margins at least $2 \mathrm{~mm}$, unicentric, no extensive intraductal component (EIC), and no nodal involvement [5]. WBI is given in 50 Gray (Gy) (2.0Gy/fraction) taking place over five weeks, while EB-PBI is given in 38.5Gy in 3.85Gy fractions BID for five days in accordance with the NSABP B-39/RTOG 0413 trial [14].

Patients are initially consulted by a radiation oncologist, followed by CT simulation and contouring prior to randomization to either WBI or EB-PBI. Costs, including labour and capital, were obtained from the cancer centre administration. Median unit costs were ascertained from hospital administration. Radiation therapist and dosimetrist unit costs were based on the Ontario Nurses' Association (ONA) Allied Health. Physicist unit costs were based on the Professional Institute of the Public Service of Canada (PIPSC). In accordance with the Consolidated Health Economic Evaluation Reporting Standards (CHEERS), this paper reports the time horizon and discount rate. The time horizon is one year with a discount rate of 0 . The one-year time horizon was chosen as the hospital budget is determined for a twelve-month period, and the impact of changes can be measured during this time frame. The discount rate of 0 was chosen as we do not expect costs to change during this single year time frame. Specific costs may change and may affect the time horizon to which this paper's conclusion can be applied.

\section{Labour}

A process flow chart was created to visualize the staff time (labour) and tasks associated with each component of the treatment process (summarized in Figure 1).

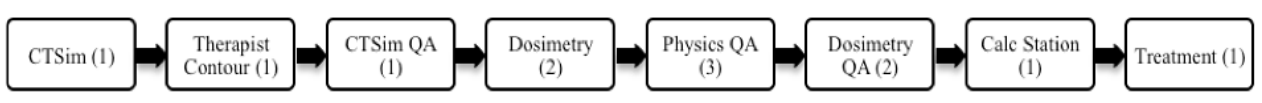

\section{FIGURE 1: Process map for patient management}

(1) Indicates radiation therapist, (2) dosimetrist, and (3) physicist responsibilities. 
This allows for costing and timing of each step in management. Detailed time stamping was obtained from all of the departments involved in the radiotherapy program, including dosimetry, physics, and radiation therapy. Individual responsibilities for each of the departments were obtained. Radiation therapist responsibilities included CT simulation, immobilization and patient marking, contouring critical structures, quality assurance and verification, and delivery of treatment. Dosimetrist responsibilities consisted of quality assurance and development of a treatment plan. Physicist duties included quality assurance and reviewing of the final plan. Specific time requirements for each responsibility was then obtained and validated. Labour times and individual responsibilities were validated through staff interviews, retrospective patient data, review of articles from literature searches, and Cancer Care Ontario (CCO) data.

\section{Capital}

Capital costs included the depreciation of the radiation therapy machine expressed in $\$$ per delivered Gy per year. Operational service costs are additional but are spread out over the entire patient workload. Standard CCO operation of a linear accelerator is 10 hours per workday. Using provincially validated parameters of 3.4 fractions delivered per hour and 20 fractions per treated case, the projected annual treated patients for one linac is 400 . The annual approximate depreciated linac cost per year is $\$ 300,000$, obtained from the 2011 cost of $\$ 3 \mathrm{M}$ for a dual energy, IMRT, VMAT, and IGRT-equipped linac divided by the expected lifetime of 10 years. The number of fractions delivered annually by one linac is approximately 8,000 . Therefore, the annual depreciated linac cost per fraction is $\$ 300,000 / 8,000=$ $\$ 40 /$ fraction. Assuming the average dose prescription is $2 \mathrm{~Gy} /$ fraction, this corresponds to a total prescribed dose of 16,000Gy. At LRCP, the annual depreciated linac cost per Gy = $\$ 300,000 / 16,000=\$ 18.75$ per Gy, further adjusted to WBI or EB-PBI per case.

\section{Results}




\section{Cureus}

WBI (hrs)

0.50

0.25

0.58

6.25

7.58

Total

Dosimetrist

Dosimetry

Dosimetry QA

Total

Physicist

Pre-randomization

Physics QA

Total
1.50

0.35

1.85

1.00

0.27

1.27
EB-PBI (hrs)

0.50

0.75

0.58

3.33

5.17

3.13

0.63

3.75

1.00

0.50

1.50

\section{TABLE 1: Summary of individual labour times}

The majority of labour time was spent on the treatment stage, with WBI and EB-PBI taking 6.25 hours and 3.33 hours, respectively. This was primarily due to the difference in number of treatments as opposed to length of individual treatments.

The costs associated with the two treatment arms are summarized in Tables 2, 3. 


\section{Cureus}

\begin{tabular}{|c|c|c|c|c|c|}
\hline & & Units (hr) & Cost (\$) & & \\
\hline & Unit cost $(\$ / \mathrm{hr})$ & WBI & EB-PBI & WBI & EB-PBI \\
\hline \multicolumn{6}{|l|}{ Labour } \\
\hline Radiation therapist & 34.22 & 7.58 & 5.17 & 259.39 & 176.92 \\
\hline Dosimetrist & 34.22 & 1.85 & 3.75 & 63.31 & 128.33 \\
\hline Medical physicist & 67.72 & 1.27 & 1.50 & 86.00 & 101.58 \\
\hline Capital (per case) & & & & WBI & EB-PBI \\
\hline Linac cost & & & & 937.5 & 721.88 \\
\hline
\end{tabular}

TABLE 2: Summary of costs

\begin{tabular}{|c|c|c|c|c|}
\hline & WBI & EB-PBI & & \\
\hline & \$ per patient & $\%$ of total cost & \$ per patient & $\%$ of total cost \\
\hline Labour (staff costs) & 408.70 & $30 \%$ & 406.82 & $36 \%$ \\
\hline Capital & 937.50 & $70 \%$ & 721.88 & $64 \%$ \\
\hline Total & $1,346.20$ & $100 \%$ & $1,128.70$ & $100 \%$ \\
\hline
\end{tabular}

\section{TABLE 3: Costs of WBI and EB-PBI Totals}

Labour accounted for $30 \%$ and 36\% of the total costs for WBI and EB-PBI, respectively. Capital accounted for $70 \%$ and $64 \%$ of the total costs for WBI and EB-PBI, respectively. The majority of the cost differences were due to the greater number of treatments for WBI compared to EB-PBI, as the cost/Gy was the same.

Table 4 shows the results of the sensitivity analysis that were carried out by varying the assumptions made regarding labour times and unit costs to calculate the maximal and minimal costs of WBI and EB-PBI, such that the differences in cost between the two treatments were the smallest or largest possible. 


\section{Cureus}

\begin{tabular}{|c|c|c|c|c|}
\hline & \multirow{2}{*}{$\begin{array}{l}\text { Least Costly Scenario (Lowest Labour } \\
\text { Times and Unit Costs) (\$) } \\
\text { WBI }\end{array}$} & \multicolumn{3}{|c|}{$\begin{array}{l}\text { Most Costly Scenario (Highest Labour } \\
\text { Times and Unit Costs) (\$) }\end{array}$} \\
\hline & & EB-PBI & WBI & EB-PBI \\
\hline Radiation therapist & 225.98 & 153.06 & 316.62 & 216.72 \\
\hline Dosimetrist & 42.78 & 101.84 & 94.53 & 170.49 \\
\hline Physicist & 67.50 & 81.00 & 105.48 & 122.49 \\
\hline Total labour cost & 336.26 & 335.90 & 516.63 & 509.70 \\
\hline Capital & 937.50 & 721.88 & 937.50 & 721.88 \\
\hline Final total cost & $1,273.76$ & $1,057.78$ & $1,454.13$ & $1,231.58$ \\
\hline $\begin{array}{l}\text { Cost difference (WBI } \\
\text { - EB-PBI) }\end{array}$ & 215.98 & & 222.55 & \\
\hline
\end{tabular}

TABLE 4: Sensitivity Analysis Results

The analysis showed that the cost differences ranged from the least costly scenario at $\$ 215.98$ to the most costly scenario at $\$ 222.55$. The least costly scenario of WBI was still more expensive than the most costly scenario of PBI by a difference of $\$ 42.18$ per patient.

\section{Discussion}

The results of this study show that the total costs of WBI and EB-PBI are 1346.20 Canadian dollars and 1128.70 Canadian dollars, respectively. While the labour costs are similar, the majority of the cost difference is due to the capital costs. The linac cost was 937.50 Canadian dollars and 721.88 Canadian dollars for WBI and EB-PBI, respectively. EB-PBI incurs a 19\% lower cost per patient compared to WBI. The cost difference per patient is $\$ 217.50$. Based on the LRCP department workload of 400 patients treated annually per linac, and site distribution numbers of $25 \%$ of patients treated with breast cancer, this would amount to 100 breast cancer patients treated annually. If EB-PBI was utilized exclusively, this would potentially provide savings of 50Gy minus $38.5 \mathrm{~Gy}=11.5 \mathrm{~Gy}$ x 100 patients $\times \$ 18.75 / \mathrm{Gy}=\$ 21,562.50$ in savings per linac per year. For nine treatment units, this would amount to savings of $\$ 194,062.50$ per year. The depreciation cost of nine linacs is approximately $\$ 300,000 \times 9=\$ 2.7 \mathrm{M}$ per year, so $\$ 194,063 / \$ 2.7 \mathrm{M}=7 \%$ of the total annual depreciation. By treating all current breast cancer patients with PBI, there is the potential for running linacs for fewer hours per week as well as reducing on the machines. This would effectively extend the lifetime of a linac by $7 \%$. In addition, with fewer patient visits if EB-PBI were utilized, this would improve patient throughput as the now unused slots could be used for other patients waiting for radiation treatments.

There were several limitations to our study. First, we assumed that the treatment outcomes, including toxicities between PBI and WBI, were equal. Unfortunately, there are few randomized trials currently available. Shah, et al. [9] concluded from a 12-year follow-up that APBI produced equivalent outcomes to WBI, including regional recurrence, disease-free survival, cause-specific survival, local recurrence, and overall survival. The ASTRO 2009 consensus 
statement discussed the possibility of an increase in the risk of IBTR, as well as an increased risk of toxicity, including local fibrosis or poor cosmesis, with APBI treatment [5]. If PBI had higher toxicities, this would possibly incur more costs due to an increase in follow-ups necessary to deal with these problems. In addition, with the stratification of patients into the three groups, "suitable", "cautionary", and "unsuitable", not all patients may be candidates for APBI. Presently, only the "suitable" group should be treated with APBI outside of a clinical trial, and the "cautionary" group requires caution and concern before being treated with APBI due to limited data [5]. However, APBI is widely utilized already without definitive evidence of equivalent efficacy and safety compared to WBI from phase III randomized clinical trials. Although we discussed the possibility of the exclusive use of PBI, it is important to note that not all patients are suitable for this technique.

Second, costs from a societal (patient) perspective were not included, as we were determining costs from a hospital point of view. Furthermore, WBI requires more treatments, so patients would have to make more visits to the cancer centre than any PBI regimen. Patients' out-ofpocket costs that were not incorporated in this analysis included prescription drugs, travel, accommodations and meals for distance travelling, parking, loss of income due to time off, homecare, family care, and homemaking [15].

Third, staff costs and labour time were specific to this centre. Labour times between PBI and WBI were identical from the initial patient consult to before dosimetry treatment planning, as our institution is still involved in the NSABP B-39/RTOG 0413 trial. With a more streamlined process and more familiarity with PBI, we predict a significant decrease in labour times and thus, costs required for that treatment arm. This specifically includes the labour times required for quality assurance after CT simulation and quality assurance for both dosimetrists and physicists. More recently, at our centre and several other institutions in Canada, scripts have been developed for PBI and utilized so routinely that more work is required to formulate WBI plans. Newer dosimetrists are trained almost exclusively in PBI development. Future cost analysis of labour times between the two techniques is definitely required.

To our knowledge, there have been no Canadian studies on the cost analysis of PBI versus WBI. Three American studies in the literature have addressed this question. Suh, et al. [12] compared several conventional whole-breast radiotherapy (RT) techniques with APBI techniques in the context of costs to both payer and patient. While hypofractionated whole breast treatment was found to be the least costly technique, APBI-3D-CRT was less expensive when compared to the conventional WBI, resulting in relative cost savings. They also determined that the majority of costs were dominated by the payer's perspective, with the patient costs contributing only a small fraction of the total societal costs.

Sher, et al. [13] developed a Markov model to compare the cost-effectiveness between EB-PBI and MS-PBI to WBI for adjuvant treatment of early-stage breast cancer. They determined that EB-PBI was the most cost-effective treatment arm over a wide range of assumptions and societal willingness-to-pay values, with the incremental cost-effectiveness ratio for WBRT compared with EB-PBI being $\$ 630,000 /$ quality-adjusted life-year. They concluded that in a cost-conscious environment, EB-PBI should be given preference over WBI to the appropriate patient.

Konski [16] performed a cost-minimization analysis between WBI and APBI only considering the cost of administering the radiation treatment. They assumed that all of the radiation treatment modalities analyzed were equally effective in preventing recurrences in breast cancer patients. It was determined that APBI-3D-CRT was less costly than conventional WBI because of fewer radiation treatments being delivered. They also hypothesized that the addition of indirect treatment costs incurred by patients (travel expenses, meal accommodations, and lost 
work) in the analysis would have increased the difference in costs between APBI and WBI.

Whelan, [17] et al. compared standard WBI of 50.0Gy in 25 fractions over 35 days versus a hypofractionated regimen of $42.5 \mathrm{~Gy}$ in 16 fractions over 22 days in women with invasive breast cancer post-breast-conserving surgery with clear resection margins and negative axillary lymph nodes. In a 10-year follow-up period, they found that hypofractionated WBI was not inferior to standard WBI for both the risk of local recurrence and a good or excellent cosmetic outcome. They further supported the use of the hypofractionated regimen in selected women for both its convenience and decreased costs compared to standard WBI. While we did not include hypofractionated WBI in our analysis, in the future we can apply our model to calculate the costs for this regimen.

\section{Conclusions}

With the potential for significant total dollar savings relative to the cost of breast cancer treatment, this study demonstrates the cost-effective argument to utilize EB-PBI in a more significant portion of breast cancer patients, where appropriate. This research demonstrates that EB-PBI minimizes costs relative to WBI in the treatment of early-stage breast cancer. These results and this model may be utilized by other centres and in other disease sites when executing decisions regarding resource allocation. Costs can be adjusted for each activity within the model, such as for changing labour times, radiotherapy fraction amounts, and unit costs. This model can be used by other centres to provide specific prediction of costs based on proposed changes in management or process.

\section{Additional Information \\ Disclosures}

Human subjects: All authors have confirmed that this study did not involve human participants or tissue. Animal subjects: All authors have confirmed that this study did not involve animal subjects or tissue. Conflicts of interest: In compliance with the ICMJE uniform disclosure form, all authors declare the following: Payment/services info: The research was funded by the Mach-Gaensslen foundation through the Summer Research Training Program at Western University Schulich School of Medicine \& Dentistry. Financial relationships: All authors have declared that they have no financial relationships at present or within the previous three years with any organizations that might have an interest in the submitted work. Other relationships: All authors have declared that there are no other relationships or activities that could appear to have influenced the submitted work.

\section{Acknowledgements}

This work was funded by the Mach-Gaensslen Foundation of Canada through the Summer Research Training Program at Schulich School of Medicine \& Dentistry.

\section{References}

1. Fisher B, Anderson S, Bryan J, et al.: Twenty-year follow-up of a randomized trial comparing total mastectomy, lumpectomy, and lumpectomy plus irradiation for the treatment of invasive breast cancer. N Engl J Med. 2002, 347:1233-41.

2. Kurer HM, Julian TB, Strom EA, et al.: Accelerated partial breast irradiation after conservative surgery for breast cancer. Ann Surg. 2004, 239:338-51.

3. Hoopes D, Kaziska D, Chapin P, et al.: Patient preferences and physician practice patterns regarding breast radiotherapy. Int J Radiat Oncol Biol Phys. 2012, 82:674-81.

4. Sanders ME, Scroggins T, Ampil FL, Li BD: Accelerated partial breast irradiation in early-stage breast cancer. J Clin Oncol. 2007, 25:996-1002. 
5. Smith B, Arthur D, Buchholz T, et al.: Accelerated partial breast irradiation consensus statement from the American Society for Radiation Oncology (ASTRO). Int J Radiat Oncol Biol Phys. 2009, 74:987-1001.

6. Ferraro DJ, Garsa AA, DeWees TA, et al.: Comparison of accelerated partial breast irradiation via multicatheter interstitial brachytherapy versus whole breast radiation. Radiat Oncol. 2012, $7: 53$.

7. Vicini FA, Arthur D, Wazer D, et al.: Limitations of the American Society of Therapeutic Radiology and Oncology Consensus Panel guidelines on the use of accelerated partial breast irradiation. Int J Radiat Oncol Biol Phys. 2011, 79:977-84.

8. Valachis A, Mauri D, Polyzos NP, Mavroudis D, Georgoulias V, Casazza G: Partial breast irradiation or whole breast radiotherapy for early breast cancer: A meta-analysis of randomized control trials. Breast J. 2010, 16:245-251.

9. Shah C, Antonucci J, Wilkinson J, et al.: Twelve-year clinical outcomes and patterns of failure with accelerated partial breast irradiation versus whole-breast irradiation: Results of a matched-pair analysis. Radiother Oncol. 2011, 100:210-4.

10. Berrang T, Olivotto I, Kim D, et al.: Three-year outcomes of a Canadian multicenter study of accelerated partial breast irradiation using conformal radiation therapy. Int J Radiat Oncol Biol Phys. 2011, 81:1220-7.

11. Kuske R: Breast brachytherapy. Hematol Oncol Clin North Am. 1999, 13:543-558.

12. Suh WW, Pierce L, Vicini F, Hayman JA: A cost comparison analysis of partial versus wholebreast irradiation after breast conserving surgery for early-stage breast cancer. Int J Radiat Oncol Biol Phys. 2005, 62:790-6.

13. Sher D, Wittenberg E, Suh WW, Taghian AG, Punglia RS: Partial breast irradiation versus whole-breast irradiation for early-stage breast cancer: A cost-effective analysis. Int J Radiat Oncol Biol Phys. 2009, 74:440-6.

14. NSABP Protocol B-39/RTOG Protocol 0413. A randomized phase III study of conventional whole breast irradiation (WBI) versus partial breast irradiation (PBI) for women with stage 0 , I, or II breast cancer. (2012). Accessed: July 17, 2012: http://rpc.mdanderson.org/rpc/credentialing/files/B39_Protocol1.pdf.

15. Longo CJ, Deber R, Fitch M, Williams AP, D’Souza D: An examination of cancer patients' monthly out-of-pocket costs in Ontario, Canada. Eur J Cancer. 2007, 16:500-7.

16. Konski: Will partial breast irradiation be a cost-effective alternative to whole breast irradiation in the treatment of early-stage breast cancer?. Commun Oncol. 2004, 1:93-7.

17. Whelan TJ, Pignol JP, Levine MN, et al.: Long-term results of hypofractionated radiation therapy for breast cancer. N Engl J Med. 2010, 362:513-20. 